\title{
Where is physical organic chemistry going?
}

\author{
John D. Roberts \\ Gates and Crellin Laboratories of Chemistry, California Institute of Technology, Pasadena, CA \\ 91125, USA
}

Biography: John D. Roberts graduated from the University of California at Los Angeles with an A. B. (hons) degree in 1941 and the $\mathrm{Ph}$. D. degree in 1944. His academic career began in 1945, as Instructor at UCLA. In 1945-1946 he was National Research Council Fellow and Instructor at Harvard. He went to MIT in 1946 as an Instructor, was Assistant Professor in 1947 and Associate Professor in 1950. In 1953 he became Professor of Organic Chemistry at Caltech and in 1972 Institute Professor of Chemistry. He is now Institute Professor of Chemistry, Emeritus and Lecturer. $\mathrm{He}$ is a member of the National Academy of Sciences (1956) and the American Philosophical Society (1974). He received the Welch Award (1990, with W. E. Doering), the National Medal of Science (1990), and the ACS Arthur C. Cope Award (1994). Since 1939 his research has been concerned with the mechanisms of organic reactions and the chemistry of small-ring compounds. His current work involves applications of nuclear magnetic resonance spectroscopy to physical organic chemistry.

Physical organic chemistry in the United States has had practitioners for over a hundred years although the idea that there should be a separate discipline called physical organic chemistry seems to have been popularized by L. P. Hammett with his publication of a book with that name in 1940 (1).In Europe, Arthur Lapworth, Christopher Ingold, Hans Meerwein, and Walter Hückel were among the early investigators who helped to advance the field. In my view, James B. Conant was enormously influential in the United States through his breadth of interests. For example, he was involved in the early structural theories of G. N. Lewis, he used his knowledge of synthesis to try to make compounds expected to have unusual properties, such as triisopropylcarbinol, he carried out path-breaking kinetic studies such as of iodide ion with organic chlorides and of semicarbazone formation, as well as making a substantial fling into what now might be called biophysical organic chemistry or physical bioorganic chemistry, an area where his brilliant student, Frank H. Westheimer, later made exemplary progress. Conant along with Hammett, F. C. Whitmore, H. J. Lucas, and their many successors produced over 60 years or so a wonderful coherence of theory and practice that has proved of enormous value in synthetic and biochemistry, as well as in materials science (1). Also no one should forget, or minimize, the extraordinary impetus that was supplied, starting around 1930, by Linus Pauling's ideas on how organic structures could be qualitatively interpreted and predicted by resonance theory (1).

However, the fact is that many problems in physical organic chemistry can be said to be solved only in principle, because while we now understand the general nature of the factors that influence various outcomes, accurate predictions of any one particular outcome may be difficult because factors with imprecisely known, but more or less comparable magnitudes, often operate in different directions to influence that outcome. Another of Conant's brilliant students, an outstanding figure in physical organic chemistry, Paul D. Bartlett of Harvard University, early on advised workers in the field to search for problems where differences between outcomes are expected to be a factor of $10^{6}$, because then the odds of having a predominance of one clearly recognizable and physically understandable factor is very favorable.

Bartlett followed his own admonition in his early career to demonstrate that a tertiary cationic carbon is most likely to be stable if the bonds connected to it lie in one plane. He did this by synthesizing apocamphanyl chloride and showing that this tertiary chloride is less than $1 / 10^{6}$ as reactive as tert-butyl chloride (2)

At room temperature a factor of $10^{6}$ in an equilibrium constant, or in ease of formation of a transition state, corresponds to a free-energy (or free-energy of activation) difference of $8.2 \mathrm{kcal} / \mathrm{mol}$. Such an energy difference is substantial, but one should not believe that once you have achieved a $10^{6}$ or more equilibrium or rate 
<smiles>CC1(C)C2CCC1(Cl)CC2</smiles>

apocamphanyl chloride<smiles>CC(C)(C)Cl</smiles>

tert-butyl chloride

factor, you have also necessarily achieved understanding or have actually isolated one dominant factor on the process in which you are interested. Cogent examples are supplied by enzyme-catalyzed reactions, where rate factors of more than $10^{6}$ are observed between catalyzed and uncatalyzed rates with both extraordinary stereo- and regiospecificity. Clear understanding of the relative importance of the various factors that allow such large differences are still unclear. Hand waving is the norm. What is clear for an enzyme-catalyzed reaction is that there are multiple interactions that contribute both to the enthalpies and entropies of the various stages of the overall process.

One might hope that the importance and nature of those interactions could be defined and understood by the study of simple model compounds, but the fact is that this is more easily said than done, it not being easy to find simple models where a single kind of influence can be studied in a way that can be significantly applied to enzyme properties or reactivities. Indeed, the more straightforward and useful approach will surely come from modifications in the amino-acid compositions of enzymes by bioengineering and particularly by the techniques introduced by Peter G. Schultz (3) that allow study of the results of substitution of wholly unnatural amino acids at critical positions in peptides and proteins.

The factors that enter into the high efficiencies of enzyme catalyzed reactions (the ones that do not involve coordination with metal atoms) must include, although not necessarily be limited to, enthalpy changes arising from hydrogen bonding, electrostatic interactions, steric hindrance, changes in solvation, and van der Waals interactions. Tending to nullify a balance of favorable enthalpy changes for formation of enzymesubstrate complexes will be entropic factors associated with diminished translational and rotational freedom.

Quite apart from enzyme structures and catalysis, in my opinion, we do not know as much as we may think we do, by virtue of knowing how they operate in principle about the relative importance of factors such as these for simple compounds, As one example, one can consider the equilibrium ratios of gauche/trans conformations of substances as simple as 1,2-disubstituted ethanes, as a function of the nature of $\mathrm{X}$ and $\mathrm{Y}$.<smiles>[Y]C1CC2CCC1C([Y])C2[X]</smiles>

gauche

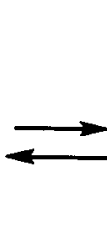

trans<smiles>[Y]C1CC2CCC1C([X])C2[X]</smiles>

gauche

Presumably, any reasonably well-informed organic chemist should be able to predict, with reasonable accuracy, the balance of factors that would determine the equilibrium ratios. Thus, if $\mathrm{X}$ and $\mathrm{Y}$ are both tertbutyl groups, steric hindrance would be large in the gauche conformations and the trans conformation should and does predominate. With other combinations of groups, the factors can operate in different directions to influence the position of equilibrium. With the halogens, for example, we might expect steric hindrance and dipole-dipole electrostatic interactions to favor trans, while van der Waals attractive forces between halogens would favor gauche. The balance depends on the particular pair of halogens involved, so usually in such cases the most we can do is to derive satisfaction by rationalizing that one influence or the other is stronger in contributing to the overall result.

When we throw in the possibility of internal hydrogen-bonding between $\mathrm{X}$ and $\mathrm{Y}$, for example as $\mathrm{OH}$ 
groups, we anticipate that gauche conformations should be favored, although now we have the possibility of solvent effects. In dilute solution in a nonprotic solvent such as $\mathrm{CCl}_{4}$, internal hydrogen bonding might be expected to be very favorable. In dimethyl sulfoxide, $\left(\mathrm{CH}_{3}\right)_{2} \mathrm{~S}=\mathrm{O}$, the oxygen is a good hydrogen-bond acceptor and could divert the $\mathrm{OH}$ groups from internal to external hydrogen bonding. Water as solvent is both a hydrogen-bond acceptor and donor and could also be expected to divert the $\mathrm{OH}$ groups from internal hydrogen bonding to external hydrogen bonding. Whatever the result, it is at best capable of being rationalized, but not yet capable of being even qualitatively predicted (4).

It may be that it is not worth the effort to insist that we do not really have a grip on physical organic chemistry until we put in a substantial effort to solve the above problems in a quantitative way. After all, we understand the principles, so why quibble about the details? I would generally agree with that, except that I feel we do need to understand solvation of organic compounds in polar solvents better than we do now. Here it should be possible to make a juncture between experiment and advanced quantum computational methods, for example those used by W. L. Jorgensen $(5,6)$ that include the effects on a solute of solvation by several hundred molecules of water.

The alternative to these sorts of perhaps "grubby"-seeming problems would be to keep looking for the kind of $10^{6}$ factors that Bartlett favored. These will always be exciting discoveries, and while there may not be a lot of them left to find in simple organic chemistry (a judgement with which George Olah would surely not agree), there is an enormous number still waiting to be uncovered and understood in bioorganic chemistry. In my view, this is where the liveliest action will be for physical organic chemistry (whatever called) in the decades to come.

\section{REFERENCES}

1. J. D. Roberts. The Chemical Intelligencer, 2, 29 (1996).

2. P. D. Bartlett and L. H. Knox. J.Am. Chem. Soc. 61, 3184 (1939).

3. Y. W. Cornish, D. Mendel and P. G. Schultz. Angew. Chem., Int. Ed. Engl. 34, 621 (1995).

4. E. Lit, F. Mallon, H. Tsai and J. D. Roberts. J. Am. Chem. Soc. 115, 9563 (1993).

5. W. L. Jorgensen and J. Tirado-Rives. Perspectives in Drug Discovery \& Design 3, 123 (1995).

6. W. L. Jorgensen, J. F. Blake, D. Lim and D. L. Severance. Trans. Faraday Soc. 90, 1727 (1994). 